\title{
Implante de Stent em Bifurcação Coronária
}

\author{
Cyro Vargues Rodrigues, André Pessanha, Valério Fuks, A ugusto N eno, N estor A ugusto C. Garcia, \\ Munir Murad
}

Rio de Janeiro, RJ

Descrevem-se dois casos de angioplastia transluminal coronária (ATC) com implante de stents em bifurcação de coronária descendente anterior (DA) com artéria diagonal (DI), destacando-se as técnicas utilizadas. No caso 1, implantou-se na DA um flexistent GianturcoRoubin (GR) e através de suas hastes, um Palmaz-Schatz (PS) no óstio de DI, com sucesso. O reestudo angiográfico em sete meses mostrou ausência de reestenose. No caso 2 , implantou-se metade de um stent PS em DA e outro PS em DI, nesta ordem, também com sucesso. No seguimento clínico de seis meses, paciente encontra-se assintomático.

\section{Stents for Bifurcational Coronary Lesion}

We describe two cases of coronary angioplasty with stents implantation in bifurcational lesion of the left anterior descending artery and the diagonal branch using new techniques successfully performed. Angiographic reestenosis was not present after seven months in one case and the patient was asymptomatic after six months of clinical follow-up in the other.

Arq Bras Cardiol, volume 69 (n' 1), 47-50, 1997

As lesões em bifurcação, quando abordadas exclusivamente pela ATC convencional por balão, mesmo com os vários tipos de técnicas hoje existentes, apresentam índices de oclusão de um dos ramos, superior a 10\%, e alta probabilidade de reestenose ${ }^{1,2}$. A utilização de implante de stents, em ambos os lados da bifurcação, tem como vantagens uma maior estabilização imediata da lesão, possibilitando uma maior expansão da luz de ambos os vasos. Descrevemos dois casos de implante de stents em bifurcações de coronária descendente anterior (DA) com a artéria diagonal (DI), utilizando duas técnicas diferentes de implante.

Caso1 - Homem de 62 anos, com quadro de angina instável, evidenciando na cinecoronariografia lesão concêntrica importante (80\%), em segmento médio de DA, na bifurcação com um longo e calibroso ramo diagonal que exibia lesão excêntrica e irregular (90\%) no óstio (fig. 1A). A coronária direita não apresentava lesões obstrutivas e o ventrículo esquerdo (VE), apenas, leve hipocinesia ânteroapical.

Foram adotadas as medicações de rotina pré procedi-

Hospital São Vicente de Paulo - Rio de Janeiro

Correspondência: Cyro Vargues Rodrigues - Hospital S. Vicente de Paulo - Rua Dr Satamini, 333 - $2^{\circ}$ - 20270-233 - Rio de Janeiro, RJ

Recebido para publicação em 26/7/96

Aceito em 28/5/97 mento e utilizado cateter-guia 8FJL4, big-max (Scimed), pela via femoral direita. Colocaram-se duas cordas guias 0,014 extra-support (ACS), uma em cada artéria, e procedida a dilatação da lesão da DI com balão 3,0x20 express supra (Scimed), obtendo-se resultado insatisfatório com discreta linha de dissecção, sem alterar o fluxo distal. Foi decidido, então, pelo implante de stent nesse local, porém, abordando primeiramente a lesão da DA, considerada como o vaso principal, em importância anatomofuncional. Optou-se por um stent tipo coil que permitisse o acesso à DI através de suas hastes. A DA foi pré dilatada com balão 3,5x20 NC express supra (Scimed) e, em seguida, implantou-se o stent GR $3,5 \times 20$. Recruzada a lesão da DI, através das hastes do stent GR, foi procedido o implante de um stent PS no óstio de DI, montado no mesmo balão utilizado na pré dilatação da lesão de DA. A expansão dos dois stents foi feita simultaneamente (fig. 1B), obtendo-se ótimo resultado angiográfico - avaliação não quantitativa - (fig. 1C). O paciente apresentou boa evolução clínica, obtendo alta hospitalar em quatro dias, utilizando, como terapêutica antiagregante, apenas AAS (200mg/dia) e ticlopidina (500mg/dia).

Após 7 meses de procedimento, paciente apresentou dor atípica, com teste funcional não conclusivo, motivando reestudo angiográfico, que mostrou discreta perda de resultado no local dos implantes, porém sem critérios para reestenose (fig. 1D).

Caso 2 - Homem de 67 anos, com quadro de angina 


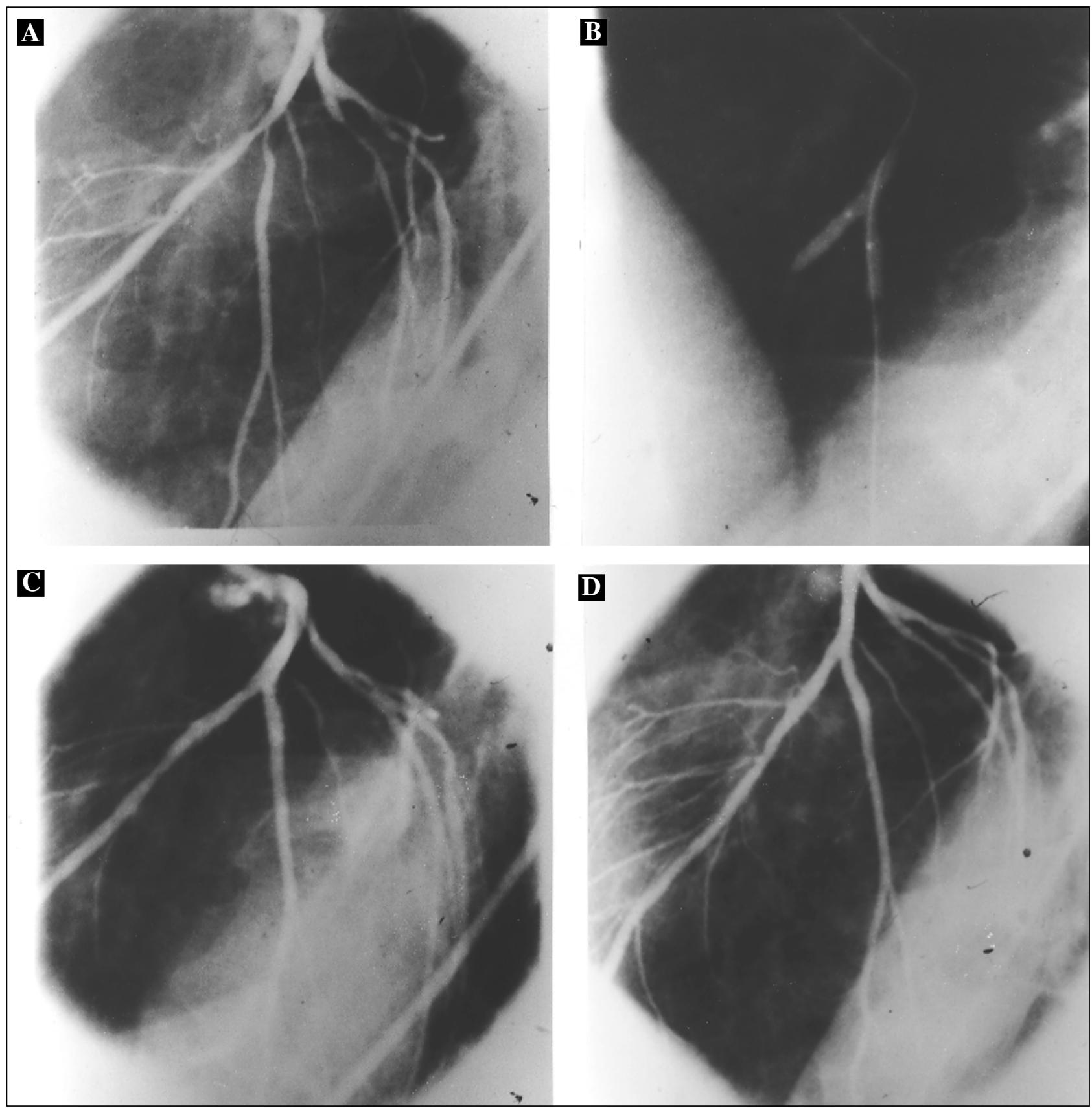

Fig. 1 - Imagens angiográficas do caso 1 - A) coronariografia esquerda em posição obliqua anterior esquerda (OAE), evidenciando lesões em bifurcação de DA e DI; B) expansão simultânea dos stents em DA e DI; C) resultado angiográfico final na mesma posição; D) reestudo angiográfico de 7 meses, com manutenção do resultado obtido em DA e DI.

aos médios esforços e teste ergométrico positivo. O estudo angiográfico evidenciou função global de VE preservada, e lesão focal concêntrica, de $90 \%$, no terço médio de DA, que apresentava segmento distal curto e com calibre reduzido, na bifurcação de uma calibrosa DI que exibia lesão segmentar, com bordos irregulares (70\%), comprometendo a bifurcação. Anatomicamente, a DI apresentava-se mais importante que a DA, mais longa e calibrosa, sendo assim considerada como o vaso principal (fig. 2A).

Após as medicações de rotina pré procedimento, pela via femoral direita, foi introduzido um cateter guia $8 \mathrm{FJL} 4 \mathrm{e}$ colocadas duas cordas guias 0,014 extra-support (ACS) respectivamente em DA e DI. Decidiu-se abordar primeiramente a DA com pré-dilatação com balão 3,0x20 (VivaScimed) e implantada metade de um stent PS montado no mesmo balão com expansão de até 18atm (fig. 2B). Em seguida, a lesão de DI com balão 3,5x20 (Viva-Scimed) foi pré-dilatada e implantado um stent PS, após a retirada da corda guia de DA. A expansão foi com o mesmo balão, com pressões de até $16 \mathrm{~atm}$. Na passagem do balão na DI, encontrouse leve resistência na altura da bifurcação, pois uma pequena parte do stent de DA tinha se projetado na luz de DI, não 
tendo sido, entretanto, fator impeditivo. Obteve-se sucesso angiográfico (análise não quantitativa ) (fig. 2C), tendo o paciente obtido alta hospitalar em dois dias, em uso de AAS $200 \mathrm{mg} /$ dia e ticlopidina $500 \mathrm{mg} / \mathrm{dia}$.

O seguimento clínico foi de seis meses, estando o paciente assintomático e com provas funcionais negativas.

\section{Discussão}

As lesões em bifurcações constituem um desafio para o cardiologista intervencionista. A ATC convencional oferece, com freqüência, resultados subótimos, com migração da placa para o outro ramo com conseqüente oclusão. Com o advento das novas técnicas intervencionistas, a aterectomia direcional para vasos com calibre superior a $2,5 \mathrm{~mm}$ com lesões não calcificadas e a aterectomia rotacional para vasos de menor calibre com lesões calcificadas, abordando ambos os vasos seqüencialmente, passaram a ser os procedimentos com resultados mais favoráveis, especialmente para atingir um luminal maior. No entanto, alguns trabalhos, não randomizados ${ }^{2,3}$, não mostraram redução dos índices de reestenose ( 40 a $50 \%$ ) e de complicações imediatas ( 3 a $10 \%$ ) na utilização dessas técnicas para esse tipo de lesão. Com os stents, consegue-se maior ganho luminal imediato, maior estabilização da lesão e menor probabilidade de oclusão do ramo. Várias técnicas de implante de stents em bifurcação têm sido descritas, como o kissing stent e outras ${ }^{4-6}$. Utilizamos, no $1^{\circ}$ caso, a técnica de implante sequiencial dos stents, iniciando pela artéria principal (DA), com stent tipo coil que permite o acesso ao outro ramo através de suas hastes, e assim o implante de outro stent, bem como manter a possibilidade de nova intervenção, em ambos os vasos, em caso de reestenose. No $2^{\circ}$ caso, utilizamos outra técnica, abordando primeiramente a lesão do vaso, funcionalmente menos importante, no caso a DA, com metade de um stent PS, por se tratar de uma lesão bastante focal, com o cuidado de não invadir a luz de DI para não impedir a abordagem da outra lesão, e, em seguida, implantamos um stent inteiro PS na DI. Esta técnica, quando utilizado apenas o stent PS, tem a desvantagem de enjaular um dos ramos, não permitindo novo acesso a esse vaso, oferecendo risco do stent implantado primeiramente no óstio do ramo, se projetar na luz do vaso principal, bloqueando a sua passagem. Por estes motivos, embora os resultados angiográficos imediatos tenham sidos semelhantes nos dois casos apresentados, achamos que a técnica utilizada no caso 1 , apresenta vantagens em relação à segunda.

Obtivemos um ótimo resultado angiográfico imediato em ambos os casos e um seguimento angiográfico (caso 1) e clínico (caso 2) bastante favoráveis.

Ainda não existem trabalhos apresentando a real diminuição dos índices de reestenose em lesões em bifurcações com as várias técnicas de implante de stents, porém, os resultados imediatos, como os ora descritos, sugerem índices bastante promissores.

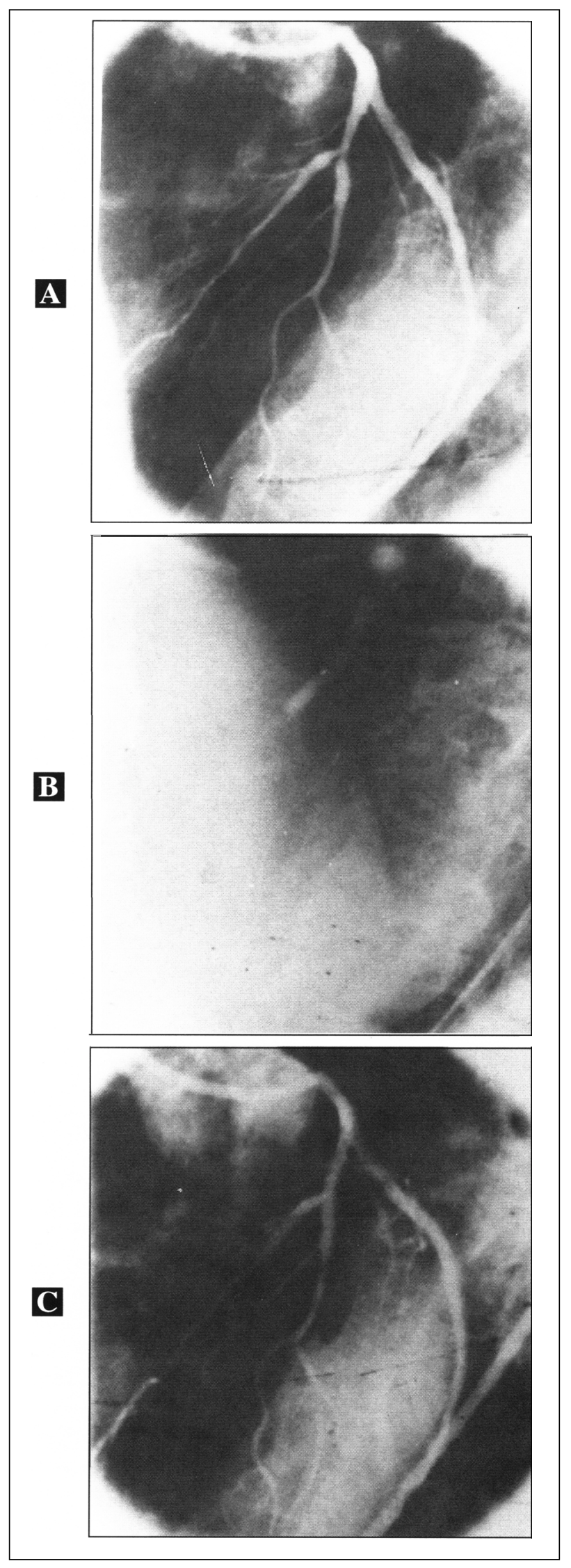

Fig. 2 - Imagens angiográficas do caso 2 - A) coronariografia esquerda em posição OAE evidenciando a importância anatômica de DI, e a lesão focal de DA e mais extensa em DI; B) implante de metade do stent $\mathrm{PS}$ em DA; C) resultado angiográfico final em OAE. 


\section{Referências}

1. Arora RR, Raymond RE, Dimas AP, Bhadwar K, Simpfendorfer C-Side branch occlusion during coronary angioplasty: incidence, angiographic characteristics and outcome. Cathet Cardiovasc Diagn 1989; 18: 210-12.

2. Mansour M, Fishman RF, Kuntz RE et al - Feasibility of directional atherectomy for the treatment of bifurcation lesions. Coronary Artery Dis 1992; 3: 761-5.

3. Warth DC, Leon MB, O'Neil W,Zacca N, Polissar NL, Bookbinder M-Rotacional atherectomy in multicenter registry: Acute results, complications and six month angiographic followup in 709 patients. J Am Coll Cardiol 1994; 24: 641-8.
4. Carrie D, Karouny E, Chouairi S, Puel J - "T"-shaped stent placement: A technique for the treatment of dissected bifurcation lesions. Cathet Cardiovasc Diagn 1996; 37: 311-13.

5. Tierstein PS - Kissing Palmaz-Schatz stents for coronary bifurcation stenoses. Cathet Cardiovasc Diagn 1996; 37: 307-10.

6. Columbo A, Gaglione A, Nakamura S, Finci L - "Kissing" stents for bifurcational coronary lesions. Cathet Cardiovasc Diagn 1993; 30: 327 30 . 